\title{
Comparison of left atrial and left atrial appendage mechanics in the risk stratification of stroke in patients with atrial fibrillation
}

Yankai Mao ${ }^{1}$, Chan Yu ${ }^{1}$ Yuan Yang ${ }^{1}$, Mingming Ma', Yunhe Wang ${ }^{2}$, Ruhong Jiang ${ }^{2}$, Ran Chen ${ }^{1}$, Bowen Zhao ${ }^{{ }^{*}}$ and Chenyang Jiang ${ }^{2^{*}}$

\begin{abstract}
Background: Left atrial (LA) and left atrial appendage (LAA) dysfunction has been demonstrated to contribute to atrial fibrillation (AF)-related stroke. However, usefulness of LA and LAA mechanics has not been fully compared. We sought to investigate the association of LA and LAA mechanics with stroke and to compare their diagnostic values in the risk stratification of stroke in patients with nonvalvular AF.

Methods: A total of 208 consecutive patients with AF (63.58 \pm 10.37 years, 63.9\% male,57.7\% persistent AF) who underwent echocardiography before catheter ablation were prospectively enrolled. Speckle-tracking was used to measure LA and LAA global longitudinal strain (GLS). LA and LAA mechanical dispersions (MD) were defined as the standard deviation (SD) of time to peak positive strain corrected by the R-R interval.

Results: Patients with prior stroke/ transient ischemic attack (TIA) $(n=31)$ had significantly higher LA and LAA MD than those without $(n=177)(11.56 \pm 4.38 \%$ vs. $8.43 \pm 3.44 \%, 15.15 \pm 5.46 \%$ vs. $10.94 \pm 4.40 \%$, both $P<0.01)$. In multivariable analysis, LA and LAA MD were independently associated with stroke/TIA (odds ratio, 1.18-1.29, 1.191.22 , respectively, both $P<0.01$ ), providing incremental values over clinical and standard echocardiographic parameters. In a subgroup analysis, LA MD was more useful than LAA MD in patients with normal LA volumes, while LAA MD was superior to LA MD in patients with LA enlargement.
\end{abstract}

Conclusions: Higher LA and LAA mechanical dispersion are independently associated with stroke/TIA in AF patients and had incremental values over clinical and conventional echocardiographic parameters. What's more, priorities of dispersion assessment are different depending on patients' LA size.

Keywords: Left atrium, Left atrial appendage, Mechanical dispersion, Speckle-tracking echocardiography, Stroke, Atrial fibrillation

\footnotetext{
* Correspondence: zbwcjp@zju.edu.cn; cyjiang@zju.edu.cn

'Department of Diagnostic Ultrasound \& Echocardiography, Sir Run Run

Shaw Hospital, Zhejiang University School of Medicine, \#3 East Qingchun Road, Hangzhou, China

${ }^{2}$ Department of Cardiology, Sir Run Run Shaw Hospital, Zhejiang University School of Medicine, 3 East Qingchun Road, Hangzhou, People's Republic of China
}

(c) The Author(s). 2021 Open Access This article is licensed under a Creative Commons Attribution 4.0 International License, which permits use, sharing, adaptation, distribution and reproduction in any medium or format, as long as you give appropriate credit to the original author(s) and the source, provide a link to the Creative Commons licence, and indicate if changes were made. The images or other third party material in this article are included in the article's Creative Commons licence, unless indicated otherwise in a credit line to the material. If material is not included in the article's Creative Commons licence and your intended use is not permitted by statutory regulation or exceeds the permitted use, you will need to obtain permission directly from the copyright holder. To view a copy of this licence, visit http://creativecommons.org/licenses/by/4.0/ The Creative Commons Public Domain Dedication waiver (http://creativecommons.org/publicdomain/zero/1.0/) applies to the data made available in this article, unless otherwise stated in a credit line to the data. 


\section{Introduction}

Non-valvular atrial fibrillation (AF) is independently associated with 5-fold increased risk of ischaemic stroke [1]. As the most devastating complication of AF, cardioembolic strokes related to AF confer increased risk of mortality and worse outcomes than non-AF strokes [2]. Currently, CHA2DS2-VASc scoring system is the widely used for estimating stroke risk in AF patient [3]. However, its direct mechanistic link with AF-related stroke has yet to be identified and its accuracy to predict ischemic stroke is modest, especially in those with a score of $<2$ [4].

AF is associated with left atrium (LA) and left atrial appendage (LAA) remodeling, which includes cavity dilation, myocardial fibrosis and subsequent dysfunction [5-7]. Although irregular contraction and subsequent intracardiac thrombosis has long been considered a direct mechanism for AF-related strokes [8], recent studies suggest that structural and functional abnormalities of LA/LAA may also contribute to stroke/ transient ischemic attack (TIA) [9-12]. LA enlargement, presence of spontaneous echo contrast (SEC) and thrombus in LA/ LAA and reduced LAA emptying velocity (LAAEV) are well-established markers of stroke risk [9, 13, 14], but stroke often occurs in patients without LA enlargement or reduced LAAEV. Speckle-tracking echocardiography (STE) provides an accurate assessment of subclinical myocardial dysfunction [15], and impaired LA and LAA global longitudinal strain (GLS) are related to stroke or embolism [12, 16-18]. Myocardial strain analysis may also measure the timing of contraction, and recent studies have demonstrated an independent association between LA and LAA mechanical dispersion (MD) and the presence of LAA thrombi or sludge [19] or stroke in AF patients [20, 21]. However, although LAA MD improved the risk stratification of embolism [21], its assessment is complicated and time-consuming. In contrast, LA MD is easier to obtain and has been validated in different clinical settings to estimate subclinical LA dysfunction and can be potentially useful for predicting a variety of cardiovascular diseases, including stroke/TIA [19, 20, 22, 23]. However, usefulness of both LA and LAA mechanics has not been fully compared for identification of stroke. This study aims to determine the associations of LA and LAA MD with stroke/TIA and to compare their incremental values in the risk stratification for stroke in patients with nonvalvular AF.

\section{Methods and materials}

\section{Study population}

We performed a cross-sectional study using 249 prospectively enrolled patients with non-valvular AF referred to Sir Run Run Shaw Hospital for catheter ablation between April 2019 and March 2020. All patients underwent preprocedural transthoracic echocardiography (TTE) and transesophageal echocardiography (TEE) with subsequent STE. We excluded 29 patients either with congenital heart disease $(n=4)$, history of any cardiac surgery and/or cardiac device implantation $(n=$ $7)$, cardiac $\operatorname{mass}(n=1)$, cardiomyopathies $(n=6)$ and apparent carotid atherosclerosis $(n=4)$, and inadequate image quality hampering strain analysis $(n=7)$. We also excluded patients who were in sinus rhythm at the time of echocardiography $(n=12)$. Patients were carefully assessed for the history of stroke or TIA. Ischemic stroke was defined by a history of hospital admission, a focal neurologic deficit of sudden onset and positive imaging findings [16]. TIA refers to neurologic signs and symptoms resolved within $24 \mathrm{~h}$ with negative imaging finding. The final population of 208 patients were classified into stroke group (with a history of stroke/ TIA before admission, $n=31$ ) and the control group $(n=177)$. The patients were classified as having either paroxysmal or persistent AF based on the guidelines [24].Clinical parameters including demographic variables, medical history and medication history were recorded. The thromboembolic risk was assessed using the $\mathrm{CHA}_{2} \mathrm{DS}_{2}$ VASc scores before stroke/TIA. The study protocol was approved by the local institutional review board and was conducted in accordance with the Declaration of Helsinki and its later amendments. All participants provided their written, informed consent.

\section{Standard echocardiography}

All patients routinely underwent TTE and TEE using a Vivid E95 echocardiograph(GE Vingmed Ultrasound AS, Horten Norway) equipped with a M5Sc (1.4-4.6 MHz) probe and a multiplane $6 \mathrm{VT}(3.0-8.0 \mathrm{MHz})$ transducer. Standard echocardiographic parameters were measured according to current recommendations [25], including left ventricular (LV) and LA volumes, LV ejection fraction (LVEF) and LA antero-posterior diameter (LAAPd). Lidocaine hydrochloride spray was used for local anaesthesia before TEE studies. A comprehensive visual assessment of LAA was performed at the mid-esophageal position by sweeping from $0^{\circ}-180^{\circ}$. The LA and LAA were examined for the presence of dense SEC or thrombus. The dense SEC was defined as very slow swirling smoke-like echoes detectable within the LA/LAA throughout the cardiac cycle. A thrombus was defined as a mobile, irregularly shaped, echo-dense mass that was clearly distinct from LA endocardium and pectinate muscles. LA volume was measured using the area-length method from the apical four and two chamber views. The LAA volume was determined using the same method from two orthogonal views typically at $45^{\circ}$ and $135^{\circ}$.The LA and LAA emptying fraction (LAEF and LAAEF) was calculated as [maximum volume $\left(\mathrm{V}_{\max }\right)$ - 
minimal volume $\left.\left(\mathrm{V}_{\min }\right)\right] / \mathrm{V}_{\max }{ }^{*} 100$. LAA emptying velocity (EV) and filling velocity (FV) was also recorded. All linear and volumetric variables were subsequently indexed to body surface area (BSA).

\section{Speckle-tracking echocardiography}

Five consecutive cardiac cycles were stored in cine-loop format for strain analysis, which was performed with vendor-dependent software (EchoPAC PC version 203, GE Vingmed Ultrasound AS, Horten Norway). We used images acquired with a frame rate of 60-80 frames/sec during breath hold. The LA endocardial border was manually traced in both four-chamber and two-chamber views. The LAA endocardium was manually traced from mid-esophageal TEE views obtained at $0^{\circ}, 45^{\circ}, 90^{\circ}$ and $135^{\circ}$. The endo-and epicardial border tracing were adjusted thereafter so that the region of interest covered full thickness of LA or LAA wall. The software divided the LA or LAA wall into 6 segments in each view and generated strain curves for each segment. Any segments that failed to track were rejected and excluded from analysis. LA or LAA GLS was obtained by averaging peak positive strain values in all segments if they were tracked adequately. LA or LAA MD was defined as the standard deviation (SD) of the time to peak positive strain of each segment and expressed as a percentage of the R-R' interval. Time-to-peaks in opposite phase to the expected direction of strains were not included in the final computation. Higher values of LA or LAA MD indicate a greater degree of LA or LAA dyssynchrony. The reference frame of zero strain was set at LV end-diastole (R$\mathrm{R}$ gating) [26]. To resolve the problem of beat-to-beat variation in STE measurements we used the index-beat method [19, 27]. Each LA or LAA measurement was estimated using the ratio of the preceding to prepreceding $\mathrm{R}-\mathrm{R}^{\prime}$ interval. All echocardiographic analysis was performed by one investigator experienced with strain imaging and blinded to the patients' information. Of the total of 4992 LAA segments and 2496 LA segments analyzed in 208 patients, STE analysis was feasible in 6968 (93.1\%) segments.

\section{Statistical analysis}

IBM SPSS package 25.0 (SPSS, Inc., Chicago, IL, USA) was used to perform the statistical analyses. Continuous data were presented as mean \pm SD. Categorical variables were expressed as numbers and percentages. Comparisons between groups were performed by using independent Student's t-test, the Mann-Whitney U test, Chisquare test or Fisher's exact test where appropriate. Univariate and multivariate binary logistic regression analysis was used to assess the associations between clinical or echocardiographic parameters and prior stroke/TIA. The independence and robustness of LA MD and LAA
MD were examined using several models. Risks were expressed as odds ratio (OR) with 95\% confidence interval (CI). The incremental values of LA MD and LAA MD over clinical characteristics and conventional echocardiographic parameters were assessed in the overall group and in subgroups (patients with normal and abnormal LA volumes). Covariate selection for model entry was based on our own hypothesis and previous findings. The incremental values of LA and LAA MD were determined by comparing the improvement in global $\chi^{2}$ value for each model. We generated receiver operating characteristic (ROC) curve to determine the ability of different variables in identifying stroke/TIA, and the area under the curve (AUC) was compared. The cut-off value was obtained using the criterion corresponding to the highest Youden index.

Inter- and intra-observer variability for LA/LAA GLS and MD were studied in 15 randomly selected patients by two independent investigators on two different occasions. Reproducibility was expressed as intra-class correlation coefficient (ICC). Statistical significance was defined as $P<0.05$.

\section{Results}

\section{Patient characteristics}

Demographic, clinical, and echocardiographic data for the study population are presented in Table 1. A total of 208 patients $(63.58 \pm 10.37$ years, $63.9 \%$ male, $57.7 \%$ persistent AF) were included in the final analysis. The stroke group were older, had higher CHA2DS2-VASc scores before stroke $(2.29 \pm 1.16$ vs $1.73 \pm 1.26, P=0.02)$, higher prevalence of LAA dense SEC or thrombi, and more frequently suffered from heart failure than the control group. In the stroke group, 11 patients (35.5\%) were on anticoagulation before stroke/TIA, while $12 \mathrm{pa}-$ tients (38.7\%) started anticoagulation after stroke/TIA, and the remaining 8 patients $(25.8 \%)$ did not use anticoagulants. Other clinical characteristics did not show significant differences between two groups.

Patients in the stroke group had higher indexed LA volumes (Vmax and Vmin), lower LAEF, depressed LA and LAA GLS, suggesting that these patients had impaired LA and LAA function compared with controls. Furthermore, the stroke group showed more pronounced LA MD $(11.56 \pm 4.38 \%$ vs $8.43 \pm 3.44 \%, P<$ $0.01)$ and LAA MD $(15.15 \pm 5.46 \%$ vs $10.94 \pm 4.40 \%, P<$ $0.01)$ than those of the control group. Figure 1 shows representative cases of LA and LAA strain curves and MD in patients with and without stroke.

\section{Factors associated with prior stroke/TIA}

The univariate logistic regression analysis identified a variety of clinical, TTE and TEE parameters as significant contributors to prior stroke/TIA. Age, LA MD and 
Table 1 Clinical and echocardiographic characteristics of the study population

\begin{tabular}{|c|c|c|c|c|}
\hline & Overall $(N=208)$ & Stroke/TIA $(N=31)$ & Control $(N=177)$ & $P$ value \\
\hline \multicolumn{5}{|l|}{ Clinical characteristics } \\
\hline Gender,male & $133(63.9)$ & $20(64.5)$ & $113(63.8)$ & 0.94 \\
\hline Age,years & $63.58 \pm 10.37$ & $68.32 \pm 7.57$ & $62.75 \pm 10.59$ & $<0.01$ \\
\hline Body mass index,kg/m² & $24.37 \pm 3.20$ & $24.72 \pm 3.07$ & $24.31 \pm 3.22$ & 0.52 \\
\hline Heart failure & $33(15.9)$ & $10(32.3)$ & $23(13.0)$ & $<0.01$ \\
\hline Coronary artery disease & $37(17.8)$ & $7(22.6)$ & $30(16.9)$ & 0.45 \\
\hline Hypertension & $111(53.4)$ & $19(61.3)$ & $92(52.0)$ & 0.34 \\
\hline Diabetes & 45 (21.6) & $8(25.8)$ & $37(20.9)$ & 0.54 \\
\hline Hyperlipoproteinemia & $58(27.9)$ & $5(16.1)$ & $53(29.9)$ & 0.11 \\
\hline Persistent AF & $120(57.7)$ & $20(64.5)$ & $100(56.5)$ & 0.40 \\
\hline Anticoagulation & $145(69.7)$ & $23(74.2)$ & $122(68.9)$ & 0.56 \\
\hline $\mathrm{CHA}_{2} \mathrm{DS}_{2}-\mathrm{VASc}$ score before stroke & $1.82 \pm 1.26$ & $2.29 \pm 1.16$ & $1.73 \pm 1.26$ & 0.02 \\
\hline \multicolumn{5}{|c|}{ Conventional echocardiographic parameters } \\
\hline iLVESV,mL/m2 & $24.18 \pm 9.40$ & $27.82 \pm 14.40$ & $23.56 \pm 8.17$ & 0.13 \\
\hline iLVEDV,mL/m2 & $66.84 \pm 14.55$ & $70.56 \pm 17.95$ & $66.21 \pm 13.85$ & 0.13 \\
\hline LVEF,\% & $64.26 \pm 8.24$ & $61.58 \pm 8.73$ & $64.72 \pm 8.09$ & 0.05 \\
\hline iLAAPd,mm/ m2 & $22.77 \pm 3.82$ & $23.48 \pm 3.72$ & $22.64 \pm 3.83$ & 0.26 \\
\hline iLAVmin,mL/m2 & $27.88 \pm 15.48$ & $34.86 \pm 18.81$ & $26.63 \pm 14.53$ & $<0.01$ \\
\hline iLAVmax, $\mathrm{mL} / \mathrm{m} 2$ & $41.67 \pm 17.08$ & $47.79 \pm 22.96$ & $40.57 \pm 15.63$ & 0.03 \\
\hline LAEF, \% & $35.2 \pm 16.00$ & $28.56 \pm 13.69$ & $36.39 \pm 16.13$ & 0.01 \\
\hline $\mathrm{LAAEV}, \mathrm{m} / \mathrm{s}$ & $0.53 \pm 0.24$ & $0.45 \pm 0.25$ & $0.54 \pm 0.23$ & 0.09 \\
\hline $\mathrm{LAAFV}, \mathrm{m} / \mathrm{s}$ & $0.53 \pm 0.20$ & $0.47 \pm 0.23$ & $0.54 \pm 0.20$ & 0.10 \\
\hline LAA dense SEC/thrombus & $36(18.5)$ & $11(40.7)$ & $25(14.9)$ & $<0.01$ \\
\hline ilAAVmin,mL/m2 & $1.89 \pm 1.58$ & $2.06 \pm 1.53$ & $1.86 \pm 1.59$ & 0.53 \\
\hline iLAAVmax,mL/m2 & $4.24 \pm 2.41$ & $4.12 \pm 2.58$ & $4.26 \pm 2.38$ & 0.77 \\
\hline LAAEF,\% & $57.30 \pm 19.83$ & $51.86 \pm 16.81$ & $58.19 \pm 20.19$ & 0.13 \\
\hline \multicolumn{5}{|l|}{ Strain echocardiographic parameters } \\
\hline LA GLS, $\%$ & $18.08 \pm 9.64$ & $13.09 \pm 7.01$ & $19.99 \pm 9.79$ & $<0.01$ \\
\hline LA MD,\% & $8.91 \pm 3.76$ & $11.56 \pm 4.38$ & $8.43 \pm 3.44$ & $<0.01$ \\
\hline LAA GLS,\% & $12.15 \pm 5.82$ & $9.01 \pm 3.12$ & $12.66 \pm 6.00$ & $<0.01$ \\
\hline LAA MD,\% & $11.53 \pm 4.78$ & $15.15 \pm 5.46$ & $10.94 \pm 4.40$ & $<0.01$ \\
\hline
\end{tabular}

Data are expressed as mean \pm SD or $\mathrm{N}(\%)$

$A F$ atrial fibrillation, EDV end-diastolic volume, ESV end-systolic volume, GLS global longitudinal strain, $i$ indexed to body surface area, $L A$ left atrium, $L A A$ left atrial appendage, LAA EF LAA emptying fraction, LAAEV LAA emptying velocity, LAAFV LAA filling velocity, LAAPd LA anteroposterior diameter, LAEF LA emptying fraction, $L V$ left ventricle, $L V E F$ left ventricular ejection fraction, MD mechanical dispersion, SEC spontaneous echo contrast, TIA transient ischaemic attack, Vmax maximal volume, Vmin minimal volume

LAA MD remained to be independent discriminators in each multivariate model with similar ORs (Age,1.06$1.08, P<0.05$; LA MD,1.18-1.29, $P<0.01$; LAA MD, 1.19-1.22, $P<0.01$ ) (Table 2).

The ROC curve analysis results are summarized in the Supplementary data Table 1. The AUC for most STE parameters were higher than clinical and standard echocardiographic variables, with LA MD and LAA MD having the highest diagnostic performance (AUC 0.724, 0.771, 95\% CI 0.666-0.777, 0.714-0.822, respectively). Using a LA MD cut-off value of $>11.47 \%$ or LAA MD cut-off value of $>12.97 \%$, patients with stroke/TIA were identified with a sensitivity of $51.61 \%, 85.19 \%$ and specificity of $82.94 \%, 66.87 \%$, respectively.

\section{Incremental value of LA MD and LAA MD in risk stratification of stroke}

ROC analysis showed that the AUCs of CHA2DS2VASc score plus one of each strain (LA GLS, LAA GLS, LA MD, LAA MD) models were significantly higher than that of the CHA2DS2-VASc score alone (Fig. 2). Moreover, we summarized the prevalence of stroke/TIA 


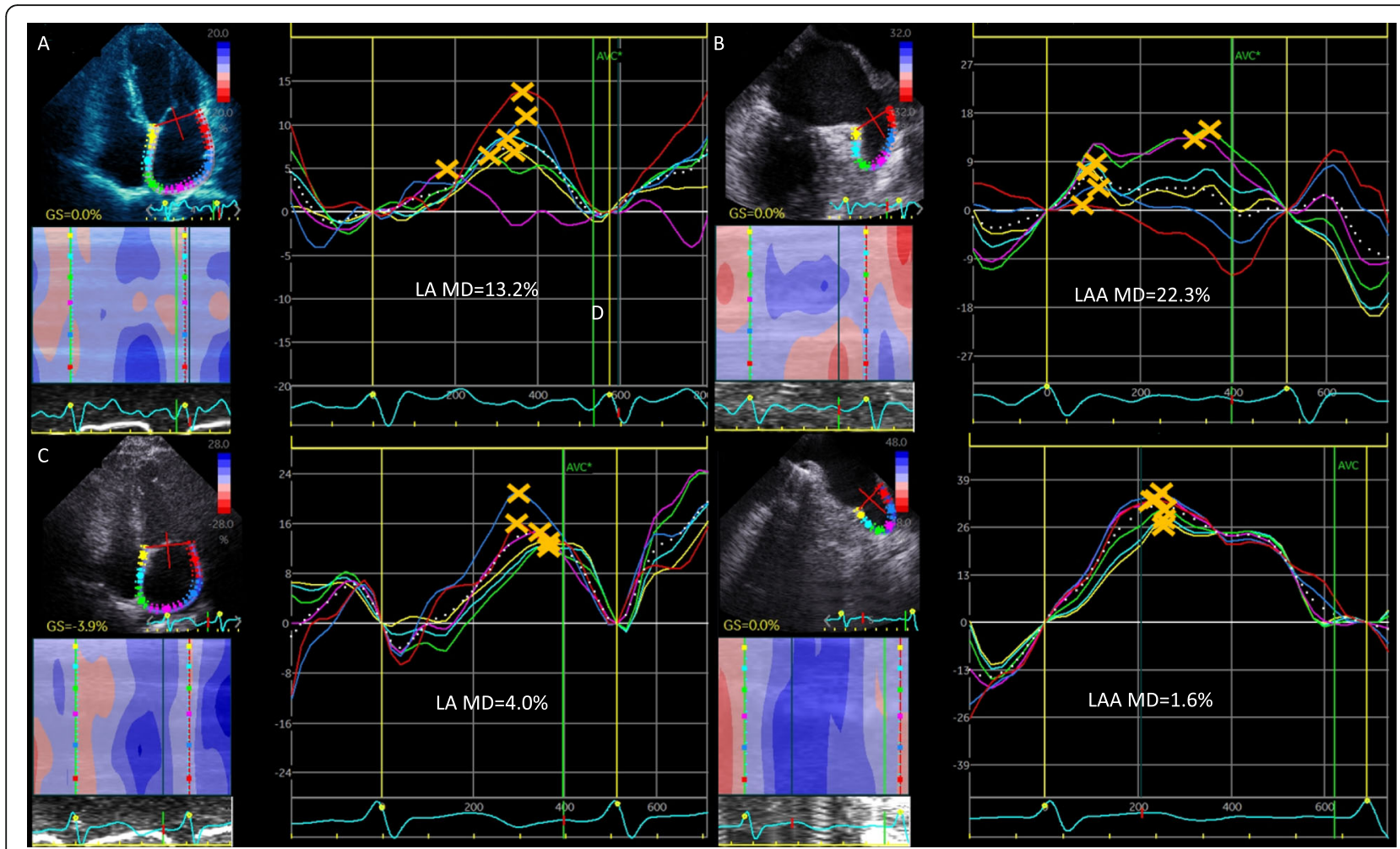

Fig. 1 LA and LAA strain curves from speckle-tracking in patients with stroke $(\mathbf{a}, \mathbf{b})$ and without stroke $(\mathbf{c}, \mathbf{d})$. Yellow crosses indicate the positive peaks of each curve. LA and LAA MD was calculated as the SD of time to peak and expressed as a percentage of the R-R interval. The patients with stroke showed higher LA and LAA MD. LA, left atrium; LAA, left atrial appendage; MD, mechanical dispersion

according to different LA MD or LAA MD and CHA2DS2-VASc score in Fig. 3. In the patients with CHA2DS2-VASc score $\geq 2(n=118)$ or $<2(n=90)$, the prevalence of stroke/TIA was significantly higher in patients with LA MD > 11.47\% (34.3\% (12/35) vs $10.8 \%$ (9/ $83), P<0.01 ; 23.5 \%(4 / 17)$ vs $8.2 \%(6 / 73), P<0.01$, respectively) or LAA MD $>12.97 \%(33.3 \%(15 / 45)$ vs $8.2 \%$ (6/73), $P<0.01$; $19.5 \%$ (8/41) vs $4.1 \%$ (2/49), $P<0.01$, respectively) than that of patients with LA MD $\leq 11.47 \%$ or LAA $\mathrm{MD} \leq 12.97 \%$. Finally, we observed the incremental benefit of LA MD or LAA MD for identifying stroke/TIA in three modeling steps. The initial model based on CHA2DS2-VASc score, iLAVmax, LAA dense SEC/Thrombus, LAAEV, LA and LAA GLS $\left(X^{2}=14.35\right)$ was significantly improved by the addition of LAA MD $\left(X^{2}=25.50, P<0.01\right)$ and further improved by adding LA $\mathrm{MD}\left(\mathrm{X}^{2}=32.41, P<0\right.$.01) (Fig. 4a). Similarly, the same initial model was also significantly improved by addition of LA MD $\left(X^{2}=23.71, P<0.01\right)$ and further improved by adding LA MD $\left(X^{2}=32.41, P<0.01\right)$ (Fig. $\left.4 b\right)$.

\section{The priorities of LA and LAA MD for risk stratification of} stroke in patients with and without LA enlargement We performed a subgroup analysis between patients with normal and abnormal LA size (Normal LA volume was defined as indexed $\mathrm{LAV}_{\max }\left(\mathrm{iLAV}_{\max }\right)<34 \mathrm{~mL} / \mathrm{m}^{2}$ [25]). Patients with LA enlargement were older, had higher prevalence of stroke and worse LA and LAA function than those with normal LA size (Supplementary data, Table 2). In the logistic regression analysis, the independent association of each variable with stroke/TIA was tested in six models (Table 3). In patients with LA enlargement, LAA MD was consistently identified as a significant contributor to previous stroke/TIA in every model but LA MD was not. Interestingly, in patients without LA enlargement, only LA MD was an independent discriminator for stroke. The incremental values of LA MD or LAA MD over CHA2DS2-VASc score, and either of LA GLS, LAEF, LAA EV, LAA GLS and LAA MD or LA MD were examined in both subgroups (Supplementary data, Figure 1). In patients with normal LA volumes, adding LA MD significantly improved all the five models, whereas, LAA MD provided no incremental value (Fig. 1a). However, only LAA MD had an additional diagnostic value for stroke in patients with LA enlargement (Fig. 1b). Furthermore, we assessed ROC analysis in both subgroups (Fig. 5). In patients with normal LA volumes, AUCs of CHA2DS2-VASc scores incorporated with both LA and LAA mechanics were significantly higher than that of CHA2DS2-VASc scores 
Table 2 Univariable and multivariable logistic regression analyses of associations between clinical and echocardiographic covariates with stroke

\begin{tabular}{|c|c|c|c|c|c|c|c|c|}
\hline \multirow[t]{3}{*}{ Variables } & \multicolumn{2}{|c|}{ Univariate analysis } & \multicolumn{6}{|c|}{ Multivariate analysis } \\
\hline & \multirow[t]{2}{*}{ OR $(95 \% \mathrm{Cl})$} & \multirow[t]{2}{*}{$P$ value } & \multicolumn{2}{|l|}{ Model 1} & \multicolumn{2}{|l|}{ Model 2} & \multicolumn{2}{|l|}{ Model 3} \\
\hline & & & OR $(95 \% \mathrm{Cl})$ & $\overline{P \text { value }}$ & OR $(95 \% \mathrm{Cl})$ & $\overline{P \text { value }}$ & OR $(95 \% \mathrm{Cl})$ & $P$ value \\
\hline \multicolumn{9}{|l|}{ Clinical parameters } \\
\hline Age & $1.07(1.02-1.12)$ & $<0.01$ & $1.06(1.01-1.13)$ & 0.03 & $1.07(1.01-1.14)$ & 0.03 & $1.08(1.01-1.40)$ & 0.02 \\
\hline Female & $1.03(0.46-2.29)$ & 0.94 & $2.17(0.79-6.02)$ & 0.14 & $2.44(0.85-7.03)$ & 0.09 & & \\
\hline Heart failure & $3.19(1.33-7.62)$ & 0.01 & $1.85(0.63-5.45)$ & 0.27 & $1.56(0.48-5.24)$ & 0.46 & & \\
\hline CHA2DS2-VASC score & $1.41(1.04-1.19)$ & 0.03 & $1.04(0.55-1.97)$ & 0.91 & $1.14(0.57-2.29)$ & 0.71 & $1.07(0.57-1.63)$ & 0.71 \\
\hline \multicolumn{9}{|l|}{ LA parameters } \\
\hline LAEF & $0.97(0.94-0.99)$ & 0.01 & $1.00(0.96-1.04)$ & 0.99 & & & & \\
\hline iLAVmax & $1.02(1.00-1.04)$ & 0.03 & $0.99(0.96-1.02)$ & 0.53 & & & $0.99(0.96-1.03)$ & 0.81 \\
\hline LA GLS & $0.91(0.86-0.97)$ & $<0.01$ & $0.99(0.90-1.09)$ & 0.88 & $0.99(0.89-1.10)$ & 0.77 & $0.99(0.89-1.09)$ & 0.78 \\
\hline LA MD & $1.25(1.22-1.39)$ & $<0.01$ & $1.20(1.07-1.36)$ & $<0.01$ & $1.29(1.11-1.49)$ & $<0.01$ & $1.18(1.03-1.34)$ & $<0.01$ \\
\hline \multicolumn{9}{|l|}{ LAA parameters } \\
\hline LAAEV & $0.18(0.02-1.33)$ & 0.09 & & & 16.81 (1.28-220.9) & 0.03 & $4.83(0.45-52.15)$ & 0.05 \\
\hline LAAEF & $0.98(0.97-1.00)$ & 0.13 & & & $1.01(0.98-1.05)$ & 0.45 & & \\
\hline LAA dense SEC/thrombus & $3.93(1.64-9.46)$ & $<0.01$ & & & $4.39(1.33-14.53)$ & 0.02 & $3.63(1.16-11.35)$ & 0.03 \\
\hline LAA GLS & $0.84(0.75-0.94)$ & $<0.01$ & $0.96(0.85-1.09)$ & 0.53 & $0.96(0.84-1.10)$ & 0.57 & $0.96(0.84-1.09)$ & 0.49 \\
\hline LAA MD & $1.21(1.09-1.32)$ & $<0.01$ & $1.19(1.07-1.33)$ & $<0.01$ & $1.22(1.08-1.38)$ & $<0.01$ & $1.21(1.08-1.36)$ & $<0.01$ \\
\hline
\end{tabular}

$A F$ atrial fibrillation, $B M I$ body mass index, $C I$ Confidence intervals, GLS global longitudinal strain, $i$ indexed to body surface area, $L A$ left atrium, $L A A$ left atrial appendage, LAAEF LAA emptying fraction, LAAEV LAA emptying velocity, LAEF LA emptying fraction, MD mechanical dispersion, OR odds ratio, SEC spontaneous echo contrast, Vmax maximal volume

(Fig. 5a). On the other hand, in patients with LA enlargement, only AUC based on CHA2DS2-VASc score and LAA MD was significantly better compared with the CHA2DVASc score alone $(0.804$ vs $0.671, P=0.018)$ (Fig. 5b).

\section{Reproducibility}

Intra-observer and inter-observer ICC were 0.984 (95\% CI 0.955-0.994) and 0.981 (95\% CI 0.945-0.993) for LA GLS, and 0.976 (95\% CI 0.933-0.992) and 0.970 (95\% CI 0.917-0.989) for LA MD; 0.967 (95\% CI 0.909-0.988) and 0.955 (95\% CI 0.879-0.984) for LAA GLS, and 0.964 (95\% CI $0.903-0.987$ ) and $0.945(95 \%$ CI $0.879-0.991$ ) for LAA MD, respectively.

\section{Discussion}

The major findings of this study were as follows: first of all, LA MD and LAA MD assessed by speckle-tracking echocardiography were independently associated with prior stroke/TIA in patients with nonvalvular AF. Secondly, both parameters provided incremental diagnostic values over clinical risk factors and conventional echocardiographic parameters. Interestingly, LA MD was more useful than LAA MD in patients with normal LA size, while LAA MD was superior to LA MD in patients with LA enlargement.
LA and LAA mechanical dispersion as biomarkers for risk stratification of stroke/TIA

Currently, there is an ongoing exploration in the mechanisms of AF-related stroke, and LA/LAA remodeling, including structural and functional alterations, have been indicated as important underlying substrates for stroke $[5,6,9-14,28]$. In addition to conventional LA parameters like LA dilation $[5,9]$, recent studies revealed that LA strain and mechanical dispersion could detect LA dysfunction in the absence of LA enlargement $[29,30]$ and had incremental diagnostic values for stroke over LA volume [16, 20, 31]. However, only one previous study [20] has linked LA MD to a history of stroke, which used tissue-tracking cardiac magnetic resonance (CMR) and was limited to patients during sinus rhythm. To the best of our knowledge, this is the first study to demonstrate the significant and independent contribution of LA MD to prior stroke/TIA using speckletracking echocardiography. LA MD > 11.47\% accurately distinguished patients at higher risk of stroke. On the other hand, despite the potential role of LAA dysfunction as a marker of stroke, previous studies failed to demonstrate independent associations between LAA strain and embolic events [18, 21]. However, we can now detect LAA asynchrony using STE, and LAA MD has incremental value for embolism risk stratification in AF patients [21]. In the present study, the optimal LAA 


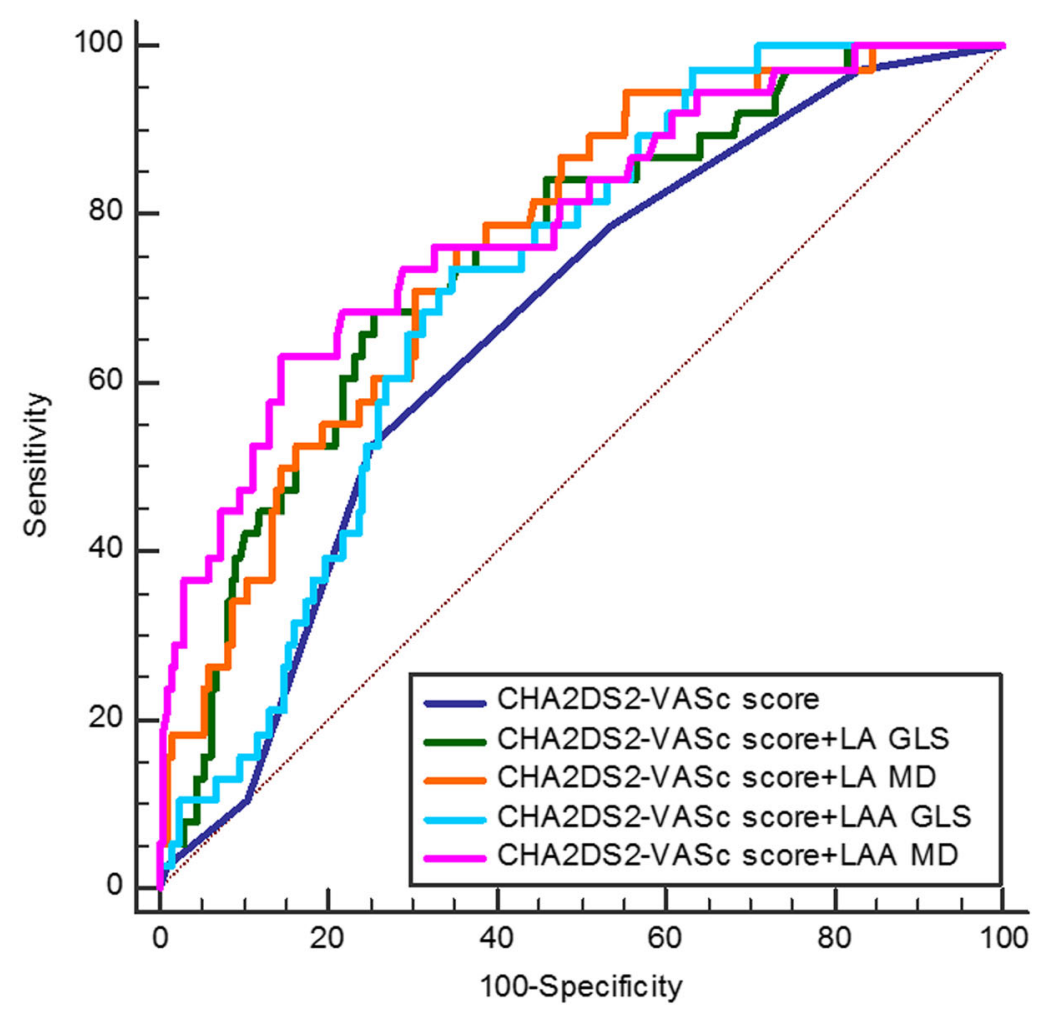

\begin{tabular}{|c|c|c|c|}
\hline & AUC & \multicolumn{2}{|c|}{$95 \% \mathrm{Cl}$} \\
\hline CHA2DS2-VASc score & 0.666 & \multicolumn{2}{|c|}{0.604 to 0.725} \\
\hline CHA2DS2-VASc score + LA GLS & 0.752 & \multicolumn{2}{|c|}{0.693 to 0.804} \\
\hline CHA2DS2-VASc score + LA MD & 0.763 & \multicolumn{2}{|c|}{0.705 to 0.815} \\
\hline CHA2DS2-VASc score + LAA GLS & 0.716 & \multicolumn{2}{|c|}{0.655 to 0.771} \\
\hline CHA2DS2-VASc score + LAA MD & 0.765 & \multicolumn{2}{|c|}{0.734 to 0.811} \\
\hline \multicolumn{3}{|c|}{ AUC comparison } & $P$ value \\
\hline \multicolumn{3}{|c|}{ CHA2DS2-VASc score vs CHA2DS2-VASc score+ LA GLS } & 0.029 \\
\hline \multicolumn{3}{|c|}{ CHA2DS2-VASc score vs CHA2DS2-VASc score+ LA MD } & 0.018 \\
\hline \multicolumn{3}{|c|}{ CHA2DS2-VASc score vs CHA2DS2-VASc score+ LAA GLS } & 0.128 \\
\hline \multicolumn{3}{|c|}{ CHA2DS2-VASc score vs CHA2DS2-VASc score+ LAA MD } & 0.015 \\
\hline
\end{tabular}

Fig. 2 Results of receiver-operating characteristic curve analysis for identifying stroke in all patients. AUC, area under the curve; GLS, global longitudinal strain; $L A$, left atrium; $L A A$, left atrial appendage; $M D$, mechanical dispersion

MD cutoff (>12.97\%) was completely consistent with our previous results [21] but in a larger sample size. We also investigated a comparison of both mechanical dispersions for the risk stratification of stroke, and identified that both variables were independent of and incremental to clinical, TTE and TEE parameters, including the other mechanical dispersion.
The mechanisms linking greater LA/LAA MD and stroke remain unclear. It is possible that inhomogenous atrial contraction may slow down the regional blood flow and lead to thrombogenesis in the LA/LAA. This is consistent with previous findings that higher atrial mechanical dispersion is associated with LAA thrombi or sludge [19, 32]. 


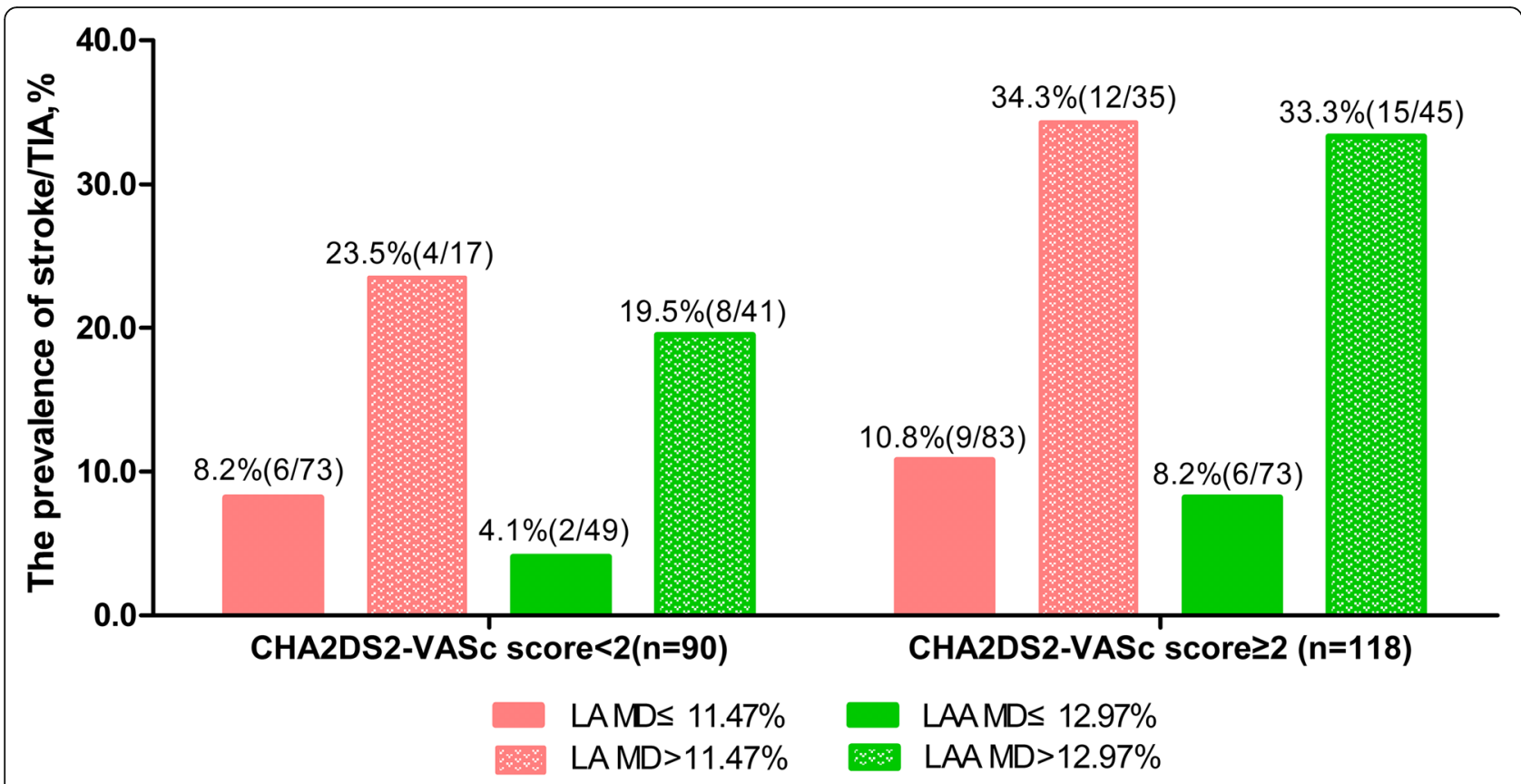

Fig. 3 The prevalence of stroke according to LA or LAA MD and CHA2DS2-VASC score. LA, left atrium; LAA, left atrial appendage; MD, mechanical dispersion

\section{Comparison of diagnostic values between LA and LAA MD}

The present study demonstrated that usefulness of LA and LAA mechanics was different depending on LA size. In patients with normal LA volumes, stroke was thought to be mainly associated with LA dysfunction or 'atrial cardiopathy' because these patients are younger and had less traditional stroke risks than those with LA enlargement. Recent evidence supported that LA MD can detect LA dysfunction and asynchrony in the absence of LA enlargement [30]. Therefore, LA MD was superior to LAA MD in patients without LA enlargement. On the other hand, only LAA MD not LA MD had a consistently significant association with a history of stroke in
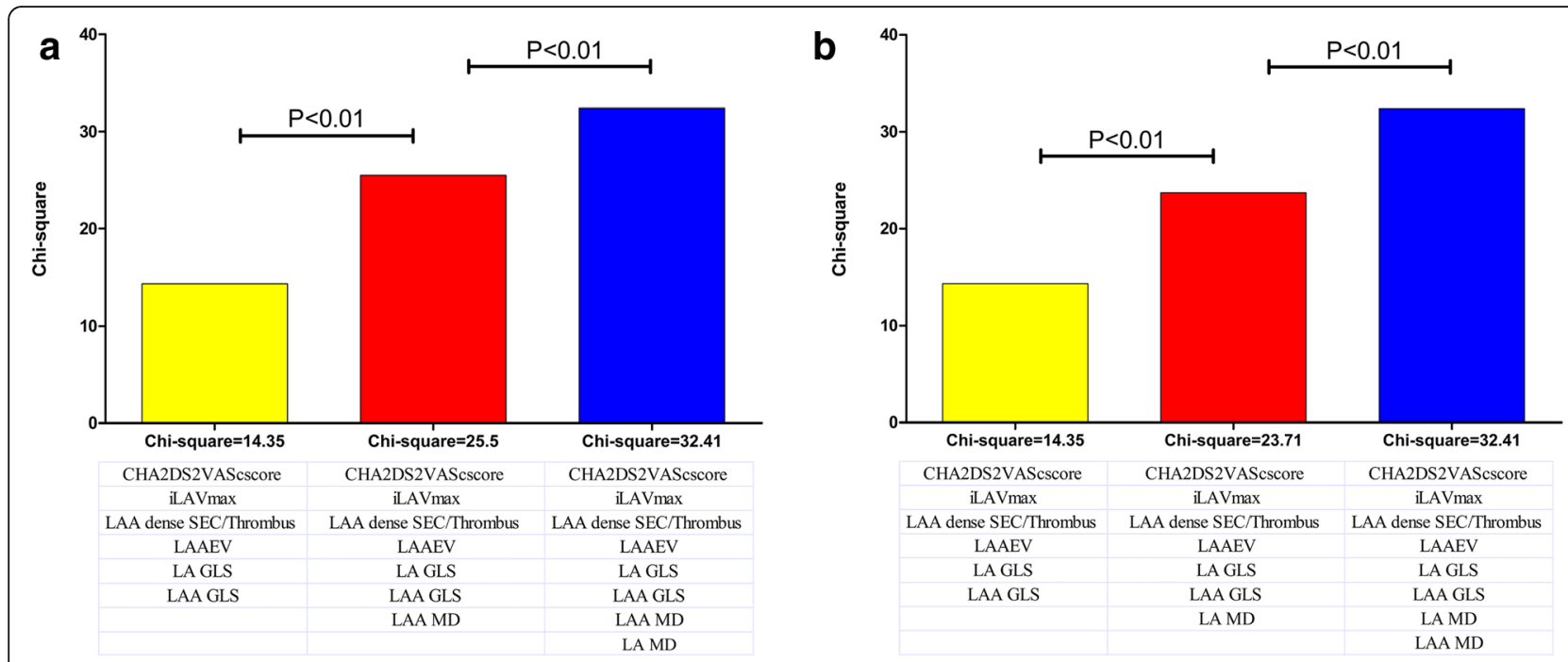

Fig. 4 Incremental values of LA and LAA MD for stroke risk stratification. a The initial model based on clinical and conventional echocardiographic parameters as well as strains was significantly improved by the addition of LA MD and further improved by adding LAA MD. $\mathbf{b}$ The same initial model was also significantly improved by the addition of LAA MD and further improved by adding LA MD. AF, atrial fibrillation; GLS, global longitudinal strain; i, indexed to body surface area; LA, left atrium; LAA, left atrial appendage; LAAEV, LAA emptying velocity; MD, mechanical dispersion; SEC, spontaneous echo contrast; Vmax, maximal volume 


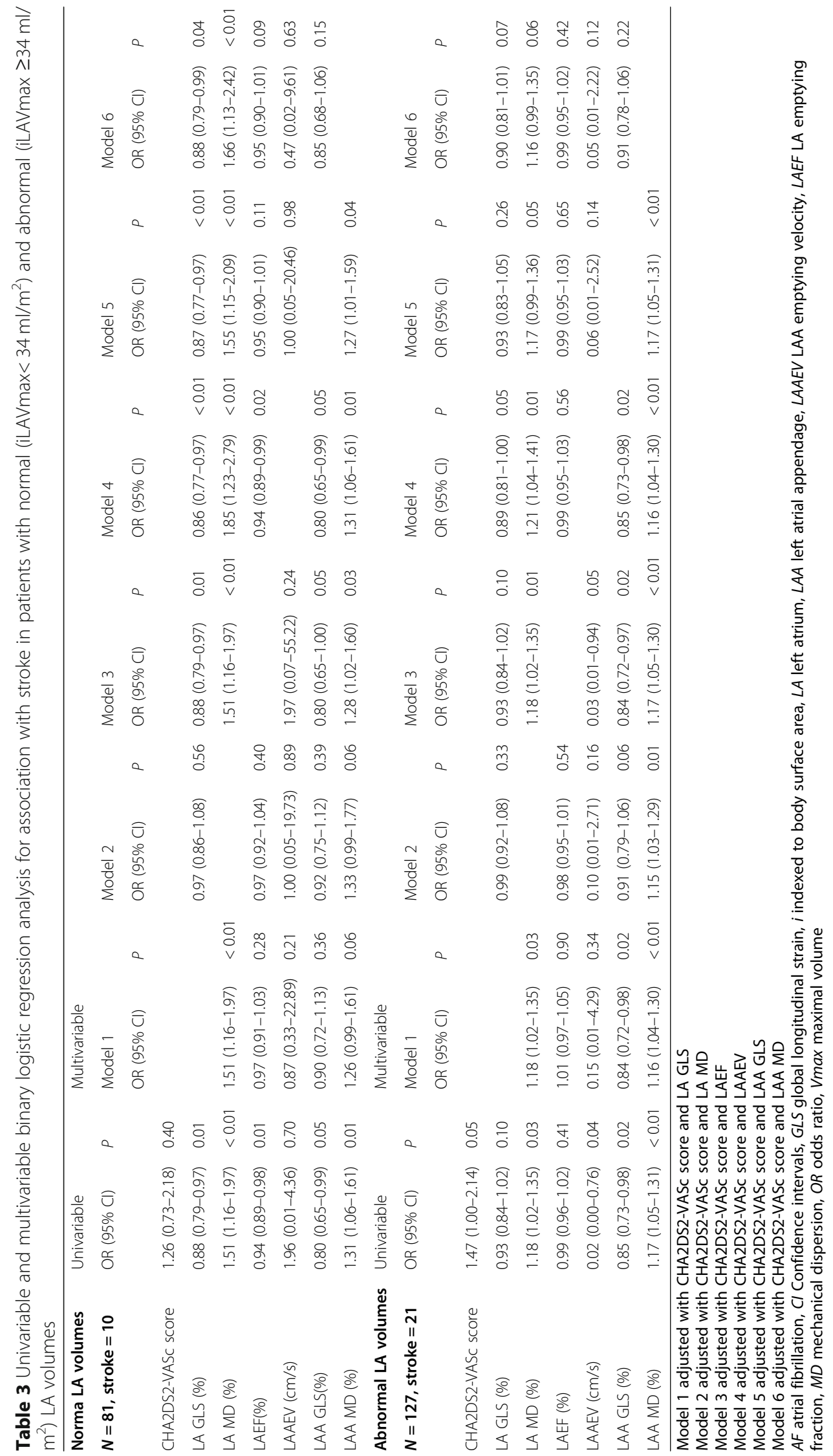



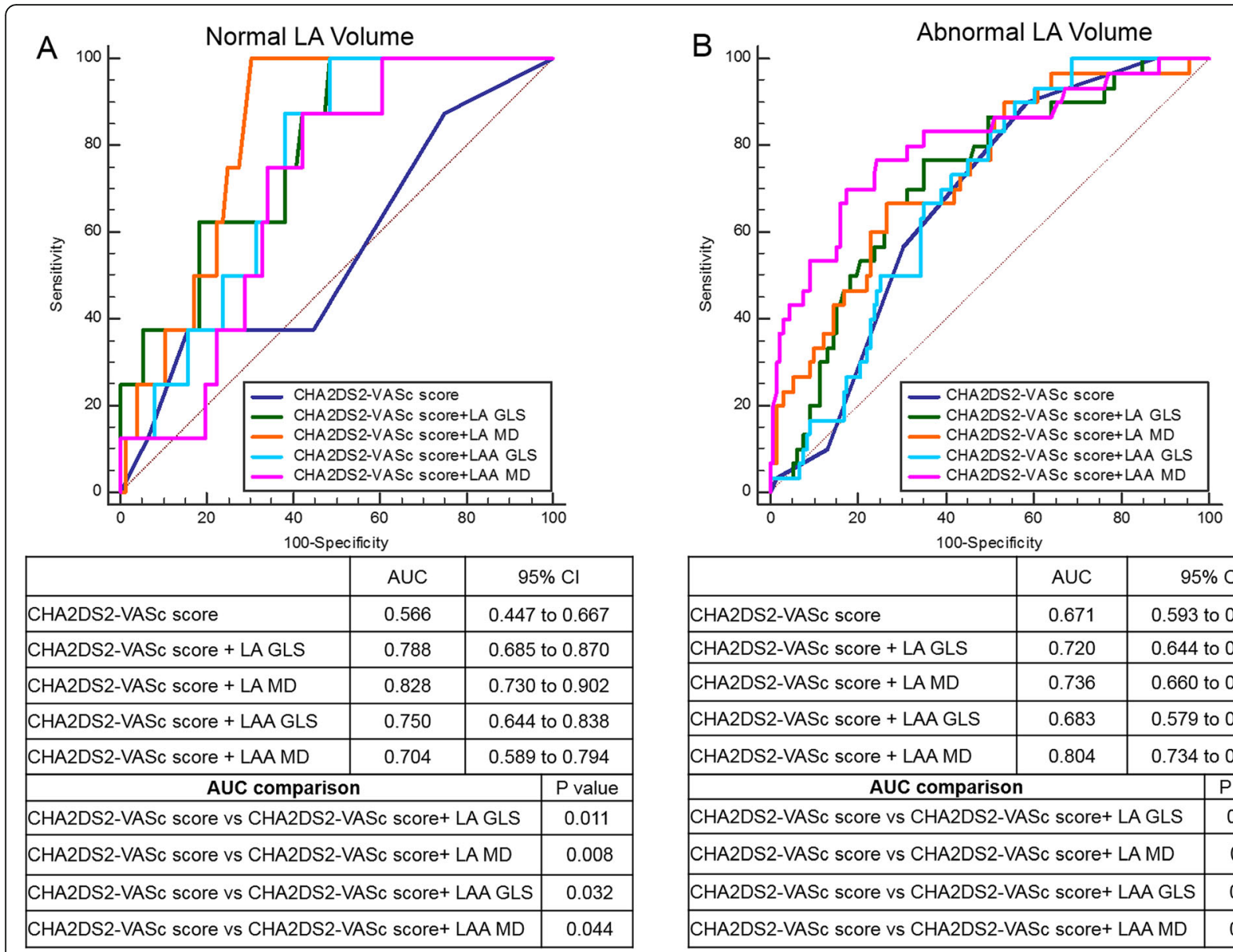

\begin{tabular}{|c|c|c|c|}
\hline & AUC & \multicolumn{2}{|c|}{$95 \% \mathrm{Cl}$} \\
\hline CHA2DS2-VASc score & 0.671 & \multicolumn{2}{|c|}{0.593 to 0.743} \\
\hline CHA2DS2-VASc score + LA GLS & 0.720 & \multicolumn{2}{|c|}{0.644 to 0.788} \\
\hline CHA2DS2-VASc score + LA MD & 0.736 & \multicolumn{2}{|c|}{0.660 to 0.802} \\
\hline CHA2DS2-VASc score + LAA GLS & 0.683 & \multicolumn{2}{|c|}{0.579 to 0.753} \\
\hline CHA2DS2-VASc score + LAA MD & 0.804 & \multicolumn{2}{|c|}{0.734 to 0.862} \\
\hline \multicolumn{3}{|c|}{ AUC comparison } & $\mathrm{P}$ value \\
\hline \multicolumn{3}{|c|}{ CHA2DS2-VASc score vs CHA2DS2-VASc score+ LA GLS } & 0.234 \\
\hline \multicolumn{3}{|c|}{ CHA2DS2-VASc score vs CHA2DS2-VASc score+ LA MD } & 0.117 \\
\hline \multicolumn{3}{|c|}{ CHA2DS2-VASc score vs CHA2DS2-VASc score+ LAA GLS } & 0.665 \\
\hline \multicolumn{3}{|c|}{ CHA2DS2-VASc score vs CHA2DS2-VASc score+ LAA MD } & 0.018 \\
\hline
\end{tabular}

Fig. 5 Results of receiver-operating characteristic curve analysis for identifying stroke in patients with normal LA volumes (iLAVmax $<34 \mathrm{~mL} / \mathrm{m}^{2}$ ) (a) and patients with abnormal LA volumes (iLAVmax $\geq 34 \mathrm{~mL} / \mathrm{m}^{2}$ ) (b). AUC, area under the curve; GLS, global longitudinal strain; i, indexed to body surface area; LA, left atrium; LAA, left atrial appendage; MD, mechanical dispersion; Vmax, maximal volume

patients with LA enlargement. In this subgroup, their LA function was significantly impaired, whereas, some non-stroke patients have preserved LAA function. That's why LAA MD could provide incremental values in patients who already had more risk factor than those with normal LA volumes.

\section{Clinical implications}

The findings in this study suggest that LA and LAA MD could be useful biomarkers to discriminate patients with strokes from controls, independent of and superior to CHA2DS2-VASc score. Therefore, assessment of LA and LAA dyssynchrony may help clinicians to identify patients at relatively higher risk for stroke. As LA/LAA remodeling are partially reversible [33, 34], therapies targeted at LA and LAA MD in addition to anticoagulation might provide benefits in patients with AF by improving LA/LAA mechanics.Future studies are warranted to test this hypothesis. In patients with LA enlargement, TEE screening can provide additional information for predicting stroke over TTE parameters. However, if the patients could not tolerate TEE or the TEE images were inadequate for strain analysis, LA MD may be an alternative to LAA MD, considering their comparable diagnostic values and greater technical difficulty in assessing LAA MD.

\section{Study limitations}

The present study has several limitations and technical considerations. First of all, this is a single-centered, cross-sectional study composed of patients with moderate to low risk for stroke. Therefore, the selection bias may influence external validity of our results. Further prospective multicenter studies are needed to confirm our findings, including the cut-off values of LA and LAA MD. Secondly, the present study recorded stroke/TIA retrospectively from the index echocardiography. Ideally baseline strain measurements should be assessed before stroke onset, but this requires a larger sample size with longer follow-up. Third, although we used multiple 
views to generate LA and LAA MD, it is still possible that these measurements were underestimated due to missing regions that were not covered by these views. Fourth, although strain imaging is operator-dependent, intra- and interobserver reproducibility was excellent in our study. Finally, we analyzed LA and LAA strain using software for evaluating the LV because dedicated atrial strain packages are not available. In addition, vendor specificity of STE should also be considered.

\section{Conclusion}

Higher LA and LAA mechanical dispersion assessed by speckle-tracking echocardiography are significantly and independently associated with a history of stroke/TIA in patients with $\mathrm{AF}$ and can provide incremental value for risk stratification of stroke over clinical and conventional echocardiographic parameters.

\section{Supplementary Information}

The online version contains supplementary material available at https:/doi. org/10.1186/s12947-020-00232-z.

\section{Additional file 1: Supplementary Table 1. AUC for ROC analysis of clinical and echocardiographic variables. The variables that have the highest diagnostic performance for stroke in each group were highlighted. Supplementary Table 2. Baseline characteristics for subgroups with normal (iLAVmax $<34 \mathrm{~mL} / \mathrm{m}^{2}$ ) and abnormal (iLAVmax $\geq 34 \mathrm{~mL} / \mathrm{m}^{2}$ ) $\mathrm{LA}$ volumes. Figure 1. The incremental value of $L A$ $M D$ or LAA MD for identifying stroke or transient ischaemic attack in six models in patients with normal LA volumes $\left.\left(i L A V_{\text {max }}\right)<34 \mathrm{~mL} / \mathrm{m}^{2}\right)(A)$ and abnormal $L A$ volumes (iLAV $V_{\max } \geq 34 \mathrm{~mL} / \mathrm{m}^{2}$ ) (B). $A F$, atrial fibrillation; $G L S$, global longitudinal strain; i, indexed to body surface area; LA, left atrium; LAA, left atrial appendage; LAAEV, LAA emptying velocity; LAEF, LA emptying fraction; $M D$, mechanical dispersion; $V_{\max }$ maximal volume}

\section{Acknowledgements}

Not applicable.

\section{Authors' contributions}

Chenyang Jiang and Bowen Zhao: Conceptualization, Methodology; Yankai Mao.: Data curation and Writing- Original draft preparation; Yuan Yang and Chan Yu:Investigation, Visualization and Formal analysis; Mingming Ma and Ran Chen: Visualization, Writing- Reviewing and Editing; Yunhe Wang: Resources and Validation. Ruhong Jiang: Resources and Funding acquisition. All authors read and approved the final manuscript.

\section{Funding}

This research was supported by the National Natural Science Foundation of China under Grant NO. 81900289.

\section{Availability of data and materials}

The datasets used and/or analysed during the current study are available from the corresponding author on reasonable request.

\section{Ethics approval and consent to participate}

The study protocol was approved by the local institutional review board and was conducted in accordance with the Declaration of Helsinki and its later amendments.

\section{Consent for publication}

All participants provided their written, informed consent.

\section{Competing interests}

The authors declare that they have no competing interests.
Received: 19 October 2020 Accepted: 10 December 2020

Published online: 09 January 2021

\section{References}

1. Virani SS, Alonso A, Benjamin EJ, Bittencourt MS, Callaway CW, Carson AP, Chamberlain AM, Chang AR, Cheng S, Delling FN, Djousse L, MSV E, Ferguson JF, Fornage M, Khan SS, Kissela BM, Knutson KL, Kwan TW Lackland DT, Lewis TT, Lichtman JH, Longenecker CT, Loop MS, Lutsey PL, Martin SS, Matsushita K, Moran AE, Mussolino ME, Perak AM, Rosamond WD, Roth GA, UKA S, Satou GM, Schroeder EB, Shah SH, Shay CM, Spartano NL, Stokes A, Tirschwell DL, LB WW, Tsao CW. Heart disease and stroke statistics2020 update: a report from the American Heart Association. Circulation. 2020;141(9):e139-596.

2. Lin HJ, Wolf PA, Kelly-Hayes M, Beiser AS, Kase CS, Benjamin EJ, D'Agostino RB. Stroke severity in atrial fibrillation. The Framingham Study. Stroke. 1996; 27:1760-4.

3. Lip GY, Nieuwlaat R, Pisters R, Lane DA, Crijns HJ. Refining clinical risk stratification for predicting stroke and thromboembolism in atrial fibrillation using a novel risk factor-based approach: the euro heart survey on atrial fibrillation. Chest. 2010;137(2):263-72.

4. van den Ham HA, Klungel OH, Singer DE Leufkens HG, van Staa TP. Comparative performance of ATRIA, CHADS2, and CHA2DS2-VASC risk scores predicting stroke in patients with atrial fibrillation: results from a national primary care database. J Am Coll Cardiol. 2015;66:1851-9.

5. Delgado V, Di Biase L, Leung M, Romero J, Tops LF, Casadei B, Marrouche N, Bax JJ. Structure and function of the left atrium and left atrial appendage: AF and stroke implications. J Am Coll Cardiol. 2017;70(25):3157-72.

6. Shirani J, Alaeddini J. Structural remodeling of the left atrial appendage in patients with chronic non-valvular atrial fibrillation: implications for thrombus formation systemic embolism, and assessment by transesophageal echocardiography. Cardiovasc Pathol. 2000;9(2):95-101.

7. Ma N, Lu R, Zhao D, Jiang Z, Tang M, Bao C, Mei J. Left atrial appendage fibrosis and 3-year clinical outcomes in atrial fibrillation after endoscopic ablation: a histologic analysis. Ann Thorac Surg. 2020 Jan;109(1):69-76.

8. Goldstein LB, Bushnell CD, Adams RJ, Appel LJ, Braun LT, Chaturvedi S, Creager MA, Culebras A, Eckel RH, Hart RG, Hinchey JA, Howard VJ, Jauch EC, Levine SR, Meschia JF, Moore WS, Nixon JV, Pearson TA. Guidelines for the primary prevention of stroke: a guideline for healthcare professionals from the american heart association/american stroke association. Stroke. 2011;42:517-84

9. Benjamin EJ, D'agostino RB, Belanger AJ, Wolf PA, Levy D. Left atrial size and the risk of stroke and death. The Framingham Heart Study. Circulation. 1995; 92:835-41.

10. Daccarett M, Badger TJ, Akoum N, Burgon NS, Mahnkopf C, Vergara G, Kholmovski E, McGann CJ, Parker D, Brachmann J, Macleod RS, Marrouche NF. Association of left atrial fibrosis detected by delayed-enhancement magnetic resonance imaging and the risk of stroke in patients with atrial fibrillation. J Am Coll Cardiol. 2011;57:831-8.

11. Ozer N, Tokgözoğlu L, Ovünç K, Kabakçi G, Aksöyek S, Aytemir K, Kes S. Left atrial appendage function in patients with cardioembolic stroke in sinus rhythm and atrial fibrillation. J Am Soc Echocardiogr. 2000;13:661-5.

12. Shih JY, Tsai WC, Huang YY, Liu YW, Lin CC, Huang YS, Tsai LM, Lin LJ. Association of decreased left atrial strain and strain rate with stroke in chronic atrial fibrillation. J Am Soc Echocardiogr. 2011;24:513-9.

13. Leung DY, Black IW, Cranney GB, Hopkins AP, Walsh WF. Prognostic implications of left atrial spontaneous echo contrast in nonvalvular atrial fibrillation. J Am Coll Cardiol. 1994;24:755-62.

14. Lee JM, Shim J, Uhm JS, Kim YJ, Lee HJ, Pak HN, Lee MH, Joung B. Impact of increased orifice size and decreased flow velocity of left atrial appendage on stroke in nonvalvular atrial fibrillation. Am J Cardiol. 2014;113:963-9.

15. Mor-Avi V, Lang RM, Badano LP, Belohlavek M, Cardim NM, Derumeaux G, Galderisi M, Marwick T, Nagueh SF, Sengupta PP, Sicari R, Smiseth OA, Smulevitz B, Takeuchi M, Thomas JD, Vannan M, Voigt JU, Zamorano JL. Current and evolving echocardiographic techniques for the quantitative evaluation of cardiac mechanics: ASE/EAE consensus statement on methodology and indications endorsed by the Japanese Society of Echocardiography. J Am Soc Echocardiogr. 2011;24:277-313.

16. Obokata M, Negishi K, Kurosawa K, Tateno R, Tange S, Arai M, Amano M, Kurabayashi M. Left atrial strain provides incremental value for embolism risk stratification over $\mathrm{CHA}_{2} \mathrm{DS}_{2}$-VASc score and indicates prognostic impact in patients with atrial fibrillation. J Am Soc Echocardiogr. 2014;27(7):709-16. 
17. Saraçoğlu E, Ural D, Kılıç S, Vuruşkan E, Şahin T, Ağaçdiken AA. Left atrial appendage 2D-strain assessed by transesophageal echocardiography is associated with thromboembolic risk in patients with atrial fibrillation. Turk Kardiyol Dern Ars. 2019;47(2):111-21.

18. Jankajova M, Kubikova L, Valocik G, Candik P, Mitro P, Kurecko M, Sabol F, Kolesar A, Kubikova M, Vachalcova M, Dvoroznakova M. Left atrial appendage strain rate is associated with documented thromboembolism in nonvalvular atrial fibrillation. Wien Klin Wochenschr. 2019:131(7-8):156-64.

19. Kupczynska K, Michalski BW, Miskowiec D, Kasprzak JD, Szymczyk E, Wejner Mik P, Lipiec P. Incremental value of left atrial mechanical dispersion over CHA2DS2 -VASC score in predicting risk of thrombus formation. Echocardiography. 2018;35(5):651-60.

20. Ciuffo L, Inoue YY, Tao S, Gucuk Ipek E, Balouch M, Lima J, Nazarian S, Spragg DD, Marine JE, Berger RD, Calkins H, Ashikaga H. Mechanical dyssynchrony of the left atrium during sinus rhythm is associated with history of stroke in patients with atrial fibrillation. Eur Heart J Cardiovasc Imaging. 2018:19(4):433-41.

21. Mao Y, Ma M, Yang Y, Yu C, Wang Y, Jiang R, Jiang C. Left atrial appendage mechanical dispersion provides incremental value for thromboembolic risk stratification over CHA2DS2-VASc score in nonvalvular atrial fibrillation. Int J Cardiol. 2020;307:41-7.

22. Kawakami H, Ramkumar S, Nolan M, Wright L, Yang H, Negishi K, Marwick $\mathrm{TH}$. Left atrial mechanical dispersion assessed by strain echocardiography as an independent predictor of new-onset atrial fibrillation: a case-control study. J Am Soc Echocardiogr. 2019;32(10):1268-1276.e3.

23. Ciuffo L, Tao S, Gucuk Ipek E, Zghaib T, Balouch M, Lima J, Nazarian S, Spragg DD, Marine JE, Berger RD, Calkins H, Ashikaga H. Intra-atrial dyssynchrony during sinus rhythm predicts recurrence after the first catheter ablation for atrial fibrillation. JACC Cardiovasc Imaging. 2019;12: 310-9.

24. January CT, Wann LS, Alpert JS, Calkins H, Cigarroa JE, Cleveland JC Jr, Conti JB, Ellinor PT, Ezekowitz MD, Field ME, Murray KT, Sacco RL, Stevenson WG, Tchou PJ, Tracy CM, Yancy CW. 2014 AHA/ACC/HRS guideline for the management of patients with atrial fibrillation: a report of the American College of Cardiology/American Heart Association task force on practice guidelines and the Heart Rhythm Society. Circulation. 2014;130:e199-267.

25. Lang RM, Badano LP, Mor-Avi V, Afilalo J, Armstrong A, Ernande L, Flachskampf FA, Foster E, Goldstein SA, Kuznetsova T, Lancellotti P, Muraru D, Picard MH, Rietzschel ER, Rudski L, Spencer KT, Tsang W, Voigt JU. Recommendations for cardiac chamber quantification by echocardiography in adults: an update from the American Society of Echocardiography and the European Association of Cardiovascular Imaging. J Am Soc Echocardiogr. 2015;28(1):1-39.e14

26. Badano LP, Kolias TJ, Muraru D, et al. Standardization of left atrial, right ventricular, and right atrial deformation imaging using two-dimensional speckle tracking echocardiography: a consensus document of the EACVI/ ASE/industry task force to standardize deformation imaging [published correction appears in Eur heart J Cardiovasc imaging. 2018 Jul 1;19(7):830833]. Eur Heart J Cardiovasc Imaging. 2018;19(6):591-600.

27. Kusunose K, Yamada H, Nishio S, Tomita N, Hotchi J, Bando M, Niki T, Yamaguchi K, Taketani Y, Iwase T, Soeki T, Wakatsuki T, Sata M. Index-beat assessment of left ventricular systolic and diastolic function during atrial fibrillation using myocardial strain and strain rate. J Am Soc Echocardiogr. 2012:25:953-9.

28. Kamel H, Okin PM, Elkind MS, ladecola C. Atrial fibrillation and mechanisms of stroke: time for a new model. Stroke. 2016;47:895-900.

29. Pathan F, Sivaraj E, Negishi K, Rafiudeen R, Pathan S, D'Elia N, Galligan J, Neilson S, Fonseca R, Marwick TH. Use of atrial strain to predict atrial fibrillation after cerebral ischemia. JACC Cardiovasc Imaging. 2018;11:1557-65.

30. Shang Z, Su D, Cong T, Sun Y, Liu Y, Chen N, Yang J. Assessment of left atrial mechanical function and synchrony in paroxysmal atrial fibrillation with two-dimensional speckle tracking echocardiography. Echocardiography. 2017;34:176-83.

31. Inoue $Y Y$, Alissa A, Khurram IM, Fukumoto K, Habibi M, Venkatesh BA, Zimmerman SL, Nazarian S, Berger RD, Calkins H, Lima JA, Ashikaga H. Quantitative tissue-tracking cardiac magnetic resonance (CMR) of left atrial deformation and the risk of stroke in patients with atrial fibrillation. J Am Heart Assoc. 2015:4:e001844.

32. Providência $\mathrm{R}$, et al. Evaluation of left atrial deformation to predict left atrial stasis in patients with non-valvular atrial fibrillation - a pilot-study. Cardiovasc Ultrasound. 2013;11:44.
33. Machino-Ohtsuka T, Seo Y, Ishizu T, Yanaka S, Nakajima H, Atsumi A, Yamamoto M, Kawamura R, Koshino Y, Machino T, Kuroki K, Yamasaki H, Igarashi M, Sekiguchi Y, Tada H, Aonuma K. Significant improvement of left atrial and left atrial appendage function after catheter ablation for persistent atrial fibrillation. Circ J. 2013;77(7):1695-704.

34. Kobayashi Y, Okura H, Kobayashi Y, Okawa K, Banba K, Hirohata A, et al. Assessment of atrial synchrony in paroxysmal atrial fibrillation and impact of pulmonary vein isolation for atrial dyssynchrony and global strain by threedimensional strain echocardiography. J Am Soc Echocardiogr. 2014;27:1193-9.

\section{Publisher's Note}

Springer Nature remains neutral with regard to jurisdictional claims in published maps and institutional affiliations.
Ready to submit your research? Choose BMC and benefit from:

- fast, convenient online submission

- thorough peer review by experienced researchers in your field

- rapid publication on acceptance

- support for research data, including large and complex data types

- gold Open Access which fosters wider collaboration and increased citations

- maximum visibility for your research: over $100 \mathrm{M}$ website views per year

At BMC, research is always in progress.

Learn more biomedcentral.com/submissions 